\title{
Extubation Readiness Practices and Barriers to Extubation in Pediatric Subjects
}

\author{
Johnny M Krasinkiewicz, Matthew L Friedman, James E Slaven, Riad Lutfi, \\ Samer Abu-Sultaneh, and Alvaro J Tori
}

\begin{abstract}
BACKGROUND: Invasive mechanical ventilation is a lifesaving intervention that is associated with short- and long-term morbidities. Extubation readiness protocols aim to decrease extubation failure rates and simultaneously shorten the duration of invasive ventilation. This study sought to analyze extubation readiness practices at one institution and identify barriers to extubation in pediatric patients who have passed an extubation readiness test (ERT). METHODS: We performed a retrospective chart review of all pediatric subjects admitted between April 2017 and March 2018, and who were on mechanical ventilation. Exclusion criteria were cardiac ICU admission, tracheostomy, chronic ventilator support, limited resuscitation status, and death before extubation attempt. Data with regard to the method of ERT and reasons for delaying extubation were collected. RESULTS: There were 427 subjects included in the analysis with $69 \%$ having had an ERT before extubation. Of those, $39 \%$ were extubated per our daily spontaneous breathing trial (DSBT) protocol, and the DSBT failed in $30 \%$ but they had passed a subsequent pressure support and CPAP trial on the same day. The most common reasons for failing the DSBT were a lack of spontaneous breathing $(30 \%$ [75/252]), being intubated $<24 \mathrm{~h}(24 \%$ [60/252]), breathing frequency outside the target range (22\% [55/252]), and not meeting tidal volume goal (14\% [34/252]). The most common documented reasons for delaying extubation despite passing DSBT were planned procedure (29\% [26/90]), neurologic status (23\% [21/90]), and no leak around the endotracheal tube (18\% [16/90]). The median time between passing ERT and extubation was $7 \mathrm{~h}$ (interquartile range, 5-10). CONCLUSIONS: In our institution, there was variation in extubation readiness practices that could lead to a significant delay in liberation from invasive ventilation. Adjustment of our DSBT to tolerate a higher work of breathing, such as higher breathing frequencies and lower tidal volumes, and incorporating sedation scoring into the protocol could be made without significantly affecting extubation failure rates. Key words: Airway extubation; extubation failure; ventilator weaning; intensive care units; pediatric. [Respir Care 0;0(0):1-•. (C) 0 Daedalus Enterprises]
\end{abstract}

\section{Introduction}

Invasive mechanical ventilation is a lifesaving intervention that is commonly used in pediatric ICUs. ${ }^{1,2}$ Pediatric critical care providers have recognized the risks associated with invasive ventilation, such as ventilator-associated pneumonia, ventilator-induced lung injury, ventilatorinduced diaphragmatic dysfunction, and exposure to seda-

\footnotetext{
The authors declare no conflicts of interest.

Correspondence: Johnny M Krasinkiewicz MB BCh BAO, Indiana University School of Medicine and Riley Hospital for Children at Indiana University Health, 705 Riley Hospital Drive, Riley Phase 2 Room 4900, Indianapolis, Indiana 46202. E-mail: jkrasink@iu.edu.
}

DOI: $10.4187 /$ respcare.08332 


\section{Extubation Practices in Pediatric Subjects}

tives and narcotics. ${ }^{3-7}$ To minimize these risks and the risks associated with extubation failure, a systematic approach to evaluate a patient's ability to independently maintain adequate gas exchange without excessive respiratory effort can help pediatric critical care providers liberate patients earlier from invasive ventilation.

Extubation failure in the pediatric ICU ranges from $5 \%$ to $15 \%$ and can lead to significant morbidity and mortality. ${ }^{8-10}$ Factors correlated with an increased risk of extubation failure include a longer duration of sedative use, longer duration of invasive ventilation, younger age, higher complexity of medical conditions, and diaphragmatic dysfunction. ${ }^{5,6,11-13}$ These factors are often used by clinicians to predict the pre-extubation risk of extubation failure, which can affect the timing of extubation. ${ }^{14}$ The most common reported cause of extubation failure in pediatric patients is upper-airway obstruction, with other causes that include respiratory insufficiency, muscular weakness, cardiac dysfunction, and neurologic impairment. ${ }^{8,13,15}$ Upper-airway obstruction is usually secondary to glottic and subglottic edema from the resultant inflammation that occurs from airway trauma during intubation or irritation from the endotracheal tube during invasive ventilation. ${ }^{16}$ As such, pediatric critical care providers may use nebulized racemic epinephrine, nebulized or intravenous steroids, high-flow nasal cannula, and heliox to avoid re-intubation. ${ }^{17}$

To mitigate these complications, published pediatric extubation readiness protocols focus on 4 elements: (1) frequent screening for eligibility to undergo a spontaneous breathing trial; (2) a spontaneous breathing trial that tests a patient's ability to maintain adequate minute ventilation and gas exchange; (3) evaluation of nonpulmonary factors that can affect extubation success (eg, hemodynamic status, pain, and sedation level), upperairway control (eg, cough and gag reflexes), fluid status, and evaluation of leak pressure around the endotracheal tube; and (4) planning for respiratory support after extubation. ${ }^{18-21}$ Although there are few published protocols, there currently are no standardized guidelines with regard to the best method to incorporate these elements into extubation protocols in the pediatric population, which can lead to wide variation in practice and delays in liberation from invasive ventilation.

The aims of this study were to describe extubation readiness practices in pediatric patients and identify barriers to extubation in subjects who have passed an extubation readiness test (ERT). We hypothesized that there is a variation in methods used to evaluate patients' readiness to be liberated from invasive ventilation in a single-center pediatric ICU based on pre-extubation risk factors for extubation failure, such as age, initial severity of illness, and duration of invasive ventilation, ${ }^{14}$ and that this variation in practice can lead to significant delays in extubation.

\section{QUICK LOOK}

\section{Current knowledge}

Invasive mechanical ventilation is associated with numerous complications, so pediatric critical care providers commonly implement protocolized testing to provide earlier liberation from invasive mechanical ventilation. Currently, there are no guidelines for pediatric ventilator liberation; this leads to variation in extubation practices and delays in extubation.

\section{What this paper contributes to our knowledge}

This paper identifies common barriers to extubation such as not passing an extubation readiness test, planned procedure, neurologic status, and absence of air leak. Changes that could be incorporated into clinicians' home institution protocols include tolerating a higher work of breathing, incorporating sedation scoring, and implementing respiratory therapist-driven extubation rounds to facilitate earlier extubation.

\section{Methods}

In this retrospective cohort study, we included all children on mechanical ventilation $<18$ y old who were admitted to the Riley Hospital for Children pediatric ICU between April 2017 and March 2018, and who were extubated. Riley Hospital is a quaternary-care children's hospital with $\sim 2,500$ pediatric ICU admission per year. Patients admitted to the cardiac ICU, patients who required a tracheostomy or chronic ventilator support, patients with limited resuscitation status, and patients who died without an extubation attempt were excluded. Patient demographics and clinical characteristics were obtained from Virtual PICU Systems (Los Angeles, California). Data with regard to which method of ERT and reasons for delaying extubation until the next calendar day despite passing an ERT were collected. Data were extracted from electronic medical records (Cerner, Kansas City, Missouri) and input into the RedCap database (Vanderbilt University, Nashville, Tennessee). The study was approved by the Indiana University Institutional Review Board.

\section{ERT}

Our daily spontaneous breathing trial (DSBT) is described in detail in our previous publication. ${ }^{18}$ In summary, the subjects were screened daily at $\sim 4: 00$ AM by bedside respiratory therapists to evaluate if they met the following criteria: no planned procedure; hemodynamically stable; no recent increase in vasoactive drips; 


\section{Extubation Practices in Pediatric Subjects}

spontaneously breathing; no recent increase in ventilator settings; $\mathrm{F}_{\mathrm{IO}_{2}}<0.5$, PEEP $\leq 6 \mathrm{~cm} \mathrm{H}_{2} \mathrm{O}$, and oxygen saturation $\geq 92 \%$; and peak inspiratory pressure $\leq 25$ $\mathrm{cm} \mathrm{H}_{2} \mathrm{O}$ when using a tidal volume of $6-8 \mathrm{~mL} / \mathrm{kg}$ in a volume-targeted mode or when achieving a tidal volume $\geq 6-8 \mathrm{~mL} / \mathrm{kg}$ for peak inspiratory pressure set to $\leq 25$ $\mathrm{cm} \mathrm{H}_{2} \mathrm{O}$ while using a pressure-targeted mode. If criteria were met, then PEEP would be reduced to $5 \mathrm{~cm} \mathrm{H}_{2} \mathrm{O}$ and $\mathrm{F}_{\mathrm{IO}_{2}}$ to 0.4 for 5-10 min. If the subject passed this preparation phase, then a pressure support (PS) CPAP trial was performed at a PS of $8 \mathrm{~cm} \mathrm{H}_{2} \mathrm{O}$ and CPAP of 5 $\mathrm{cm} \mathrm{H}_{2} \mathrm{O}$ for $2 \mathrm{~h}$. If the subject did not pass the DSBT PS/CPAP trial, the reason for trial failure was documented by the respiratory therapist in electronic medical records and the attending physician on service could do a subsequent PS/CPAP trial later that day after optimizations in sedation or fluid status were made. The PS values used for the subsequent trial varied based on physician judgment and ranged from 5 to $10 \mathrm{~cm} \mathrm{H}_{2} \mathrm{O}$, with CPAP ranging from 5 to $6 \mathrm{~cm} \mathrm{H}_{2} \mathrm{O}$.

\section{Definitions}

Extubation failure was defined as re-intubation within 48 $\mathrm{h}$ after the first extubation attempt. Extubating according to our DSBT protocol included all subjects extubated after passing both the preparation phase and a 2-h PS/CPAP trial of $8 / 5 \mathrm{~cm} \mathrm{H}_{2} \mathrm{O}$ for $2 \mathrm{~h}$. Extubating "off protocol" was defined as extubating subjects who either did not receive any form of ERT or had failed any phase of the DSBT protocol but passed a subsequent PS/CPAP trial on the same calendar day.

\section{Statistical Analysis}

Data were exported from the RedCap database and then analyzed by using SAS v9.4 (SAS Institute, Cary, North Carolina). The duration of invasive mechanical ventilation was divided into 4 categories: $<24 \mathrm{~h}, 1-7 \mathrm{~d}, 8-14 \mathrm{~d}$, and $>14$ d. Continuous variables were reported as medians with 25th, 75th interquartile range (IQR), and categorical variables were reported as frequencies and percentages. The Kruskal-Wallis test was used for continuous variables, and the chi-square test or the Fisher exact test was used for categorical variables, as appropriate. Multivariate analyses were performed by using variables that were significant at $P<.20$ in the bivariate models as well as any demographic and clinical variables deemed relevant. All statistical analyses were made by considering a significance level of $P<.05$.

\section{Results}

Between April 2017 and March 2018, there were 705 patients admitted to our pediatric ICU who required invasive ventilation. A total of 278 patients were excluded: 231 had a tracheostomy or were on chronic ventilator support, 35 patients had limited code status, and 12 patients died without an extubation attempt. The remaining 427 patients who met inclusion criteria had a median age of 40 months (IQR 9-134). Respiratory etiology was the most common primary illness category $(38.6 \%$ [165/427]), followed by injury and/or poisoning (19.0\% [81/427]), and neurologic etiology (14.5\% [62/427]). Subject demographics and clinical characteristics are summarized in Table 1.

\section{ERT Method}

Before extubation, $69.3 \%$ of the subjects (296/427) had an ERT; with $39.4 \%$ of the subjects (168/427) extubated after passing a DSBT and 30.0\% (128/427) extubated after failing a DSBT but passing a subsequent PS/CPAP trial on the same day (Fig. 1). No ERT was done before extubation in $29.0 \%$ of the subjects (124/427), and $1.6 \%$ of extubations (7/427) were unplanned. The subjects who did not receive any ERT were older; had a lower PRISM (pediatric risk of mortality score) III score; and had been admitted to the pediatric ICU for an injury, poisoning, or neurologic etiology, or after surgery (Table 1). Of the subjects intubated $<24 \mathrm{~h}$, $57.6 \%(80 / 139)$ were extubated without a formal ERT, whereas, $63.6 \%(35 / 55)$ and $70.6 \%(12 / 17)$ of the subjects intubated for 8-14 d and >14 d, respectively, were extubated according to our DSBT protocol (Table 2).

The reasons for extubating subjects off our DSBT pro-tocol are summarized in Figure 2. Of the subjects who were extubated "off protocol," a lack of spontaneous breathing (29.8\% [75/252]), intubation < $24 \mathrm{~h}(23.8 \%$

[60/252]), breathing frequency outside the target range (21.8\% [55/252]), and peak inspiration pressure or tidal volume goal not met (13.5\% [34/252]) were the most common documented reasons. Reasons for extubating off protocol could fall into $>1$ category, and the subjects had between 1 and 4 documented reasons, with a median of 1 (IQR 1-2).

\section{Barriers to Extubation}

Reasons for delaying extubation despite passing a DSBT are summarized in Figure 3. The most common reasons were a planned procedure (28.9\% [26/90]), neurologic status $(23.3 \%$ [21/90]), and no leak around the endotracheal tube $(17.8 \%$ [16/90]). Reasons for delaying extubation could fall into $>1$ category. The subjects had between 1 and 3 documented reasons, with a median of 1 (IQR 1-2). On the day of extubation, the median time between passing an ERT (DSBT or PS/CPAP) and extubation was $7 \mathrm{~h}$ (IQR $5-10)$, regardless of the duration of invasive ventilation (Table 2). 


\section{Extubation Practices in Pediatric Subjects}

Table 1. Subject Demographics and Clinical Characteristics by Extubation Readiness Test Method

\begin{tabular}{|c|c|c|c|c|c|c|}
\hline Variable & $\begin{array}{l}\text { All Subjects } \\
(N=427)\end{array}$ & $\begin{array}{c}\text { DSBT } \\
(n=168)\end{array}$ & $\begin{array}{l}\mathrm{PS} / \mathrm{CPAP} \\
(n=128)\end{array}$ & $\begin{array}{l}\text { No ERT } \\
(n=124)\end{array}$ & $\begin{array}{l}\text { Unplanned Extubation } \\
\qquad(n=7)\end{array}$ & $P$ \\
\hline Age, median (IQR) mo & $40(9-134)$ & $3.5(6-94)$ & $58.5(10-145.5)$ & $65(15.5-163)$ & $37(4-188)$ & .02 \\
\hline Age categories, $n(\%)$ & & & & & & .058 \\
\hline $0-12 \mathrm{mo}$ & $123(28.8)$ & $57(33.9)$ & $35(27.3)$ & $29(23.4)$ & $2(28.6)$ & \\
\hline $1-5 y$ & $116(27.2)$ & $53(31.6)$ & $29(22.7)$ & $32(25.8)$ & $2(28.6)$ & \\
\hline $6-11$ y & $80(18.7)$ & $31(18.5)$ & $26(20.3)$ & $23(18.6)$ & $0(0)$ & \\
\hline$>11 \mathrm{y}$ & $108(25.3)$ & $27(16.1)$ & $38(29.7)$ & $40(32.3)$ & $3(42.9)$ & \\
\hline Girls, $n(\%)$ & $192(45.0)$ & $73(43.5)$ & $56(43.8)$ & $61(49.2)$ & $2(28.6)$ & .60 \\
\hline Race/ethnicity, $n(\%)$ & & & & & & .40 \\
\hline White & $314(73.5)$ & $119(70.8)$ & $95(74.2)$ & $95(76.6)$ & $5(71.4)$ & \\
\hline African American & $75(17.6)$ & $29(17.3)$ & $23(18.0)$ & $22(17.7)$ & $1(14.3)$ & \\
\hline Hispanic & $22(5.2)$ & $14(8.3)$ & $6(4.7)$ & $2(1.6)$ & $0(0)$ & \\
\hline Other & $16(3.8)$ & $6(3.6)$ & $4(3.1)$ & $5(4.0)$ & $1(14.3)$ & \\
\hline PRISM III score, median (IQR) & $3(0-7)$ & $4(0-8)$ & $3.5(0-7)$ & $2(0-5)$ & $5(3-10)$ & .009 \\
\hline \multicolumn{6}{|l|}{ Primary illness category, $n(\%)$} & .005 \\
\hline Respiratory & $165(38.6)$ & $77(45.8)$ & $48(37.5)$ & $38(30.7)$ & $2(28.6)$ & \\
\hline Injury and/or poisoning & $81(19.0)$ & $25(14.9)$ & $22(17.8)$ & $33(26.6)$ & $1(14.3)$ & \\
\hline Neurologic & $62(14.5)$ & $16(9.5)$ & $16(12.5)$ & $28(22.6)$ & $2(28.6)$ & \\
\hline Infectious & $48(11.2)$ & $25(14.9)$ & $16(12.5)$ & $5(4.0)$ & $2(28.6)$ & \\
\hline Hematology and/or oncology & $28(6.6)$ & $11(6.6)$ & $10(7.8)$ & $7(5.7)$ & $0(0)$ & \\
\hline Other & $43(10.1)$ & $14(8.3)$ & $16(12.5)$ & $13(1.5)$ & $0(0)$ & \\
\hline Postoperative status, $n(\%)$ & $117(27.4)$ & $35(20.8)$ & $40(31.3)$ & $42(33.9)$ & $0(0)$ & .02 \\
\hline Trauma status, $n(\%)$ & $55(12.9)$ & $16(9.5)$ & $20(15.6)$ & $19(15.3)$ & $0(0)$ & .24 \\
\hline Trisomy 21, $n(\%)$ & $7(1.6)$ & $2(1.2)$ & $2(1.6)$ & $3(2.4)$ & $0(0)$ & .85 \\
\hline Bronchopulmonary dysplasia, $n(\%)$ & $16(3.8)$ & $6(3.6)$ & $6(4.7)$ & $4(3.2)$ & $0(0)$ & .87 \\
\hline Cerebral palsy, $n(\%)$ & $21(4.9)$ & $6(3.6)$ & $7(5.5)$ & $6(4.8)$ & $2(28.6)$ & .09 \\
\hline \multicolumn{7}{|l|}{$\begin{array}{l}\text { DSBT = daily spontaneous breathing trial } \\
\text { PS = pressure support } \\
\text { ERT = extubation readiness test } \\
\text { IQR = interquartile range (25th-75th) } \\
\text { PRISM = pediatric risk of mortality score }\end{array}$} \\
\hline
\end{tabular}

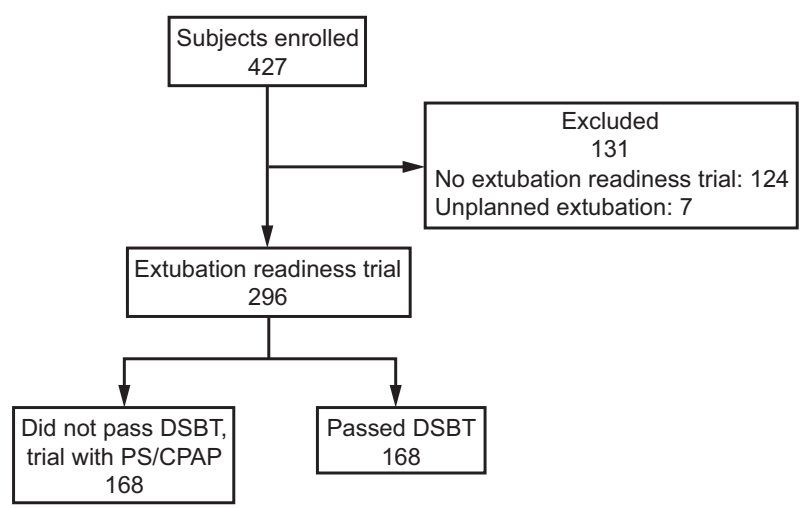

Fig. 1. Flow chart. DSBT = daily spontaneous breathing trial; $\mathrm{PS}=$ pressure support.

\section{Extubation Outcomes}

The extubation failure rate for the total cohort was $4.9 \%$ (21/427). Extubation failure rates were 8.9\% (15/168) for those who passed a DSBT, $3.1 \%(4 / 128)$ for those who failed a DSBT but passed a subsequent PS/CPAP trial,
0/124 for those who had no ERT, and $28.6 \%$ (2/7) for those who had an unplanned extubation $(P<.001)$ (Table 3$)$. Multivariate analysis demonstrated that a respiratory etiol-ogy was associated with higher odds of extubation failure (odds ratio [OR] 8.96, 95\% CI 2.49-32.27; $P<.001$ ) (Table 4). DSBT as a method of ERT did not increase the odds of extubation failure when compared with PS/CPAP (OR 0.33, 95\% CI 0.10-1.12), no ERT, or unplanned extubation (OR 7.10, 95\% CI 0.56-4.77); $P=.37$ (Table 4).

Noninvasive ventilation was used as an initial respiratory support modality after extubation in $5.4 \%$ of the subjects (23/427) for the whole cohort, with noninvasive ventilation accounting for $6.0 \%$ of the DSBT group (10/168), $4.7 \%$ of the PS/CPAP group (6/128), 5.7\% of the group that did not receive ERT $(7 / 124)$, and $0 \%$ of the unplanned extubation group $(0 / 7)(P<.001)$ (Table 3$)$. High-flow nasal cannula was used as an initial respiratory support modality in $30.9 \%$ of the subjects for the whole cohort (132/427), with highflow nasal cannula accounting for $40.5 \%$ of the DSBT group (68/168), 33.6\% of the PS/CPAP group (43/128), $13.7 \%$ of the group that did not receive ERT (17/124), and 


\section{Extubation Practices in Pediatric Subjects}

Table 2. Extubation Readiness Testing Pactices Per ilvasive Mechanical Ventilation Duration

\begin{tabular}{|c|c|c|c|c|c|c|}
\hline \multirow{2}{*}{ Variable } & \multirow{2}{*}{$\begin{array}{l}\text { All Subjects } \\
(N=427)\end{array}$} & \multicolumn{4}{|c|}{ Invasive Ventilation } & \multirow{2}{*}{$P$} \\
\hline & & $<24 \mathrm{~h}(n=139)$ & $1-7 \mathrm{~d}(n=216)$ & $8-14 \mathrm{~d}(n=55)$ & $>14 \mathrm{~d}(n=17)$ & \\
\hline ERT method & & & & & & $<.001$ \\
\hline DSBT & $168(39.3)$ & $16(11.5)$ & 105 (48.6) & $35(63.6)$ & 12 (70.6) & \\
\hline PS/CPAP & $128(30.0)$ & $41(29.5)$ & $68(31.5)$ & $16(29.1)$ & $3(17.7)$ & \\
\hline No ERT & $124(29.0)$ & $80(57.6)$ & $38(17.6)$ & $4(7.3)$ & $2(11.8)$ & \\
\hline Unplanned extubation & 7 (1.6) & $2(1.4)$ & $5(2.3)$ & $0(0)$ & $0(0)$ & \\
\hline $\begin{array}{l}\text { No. of passed DSBTs done before } \\
\text { extubation, median (IQR) [range] }\end{array}$ & $1(1-1)[1-8]$ & $1(1-1)[1-1]$ & $1(1-1)[1-6]$ & $1(1-2)[1-3]$ & $1(1-4)[1-8]$ & $<.001$ \\
\hline $\begin{array}{r}\text { Time between passing ERT and } \\
\text { extubation, median (IQR) } \mathrm{h}\end{array}$ & $7(5-10)$ & $7(5-10)$ & $7(5-10)$ & $8(6-10)$ & $7(5-11)$ & .79 \\
\hline $\begin{array}{l}\mathrm{DSBT}=\text { daily spontaneous breathing trial } \\
\text { ERT = extubation readiness test } \\
\text { PS = pressure support }\end{array}$ & & & & & & \\
\hline
\end{tabular}

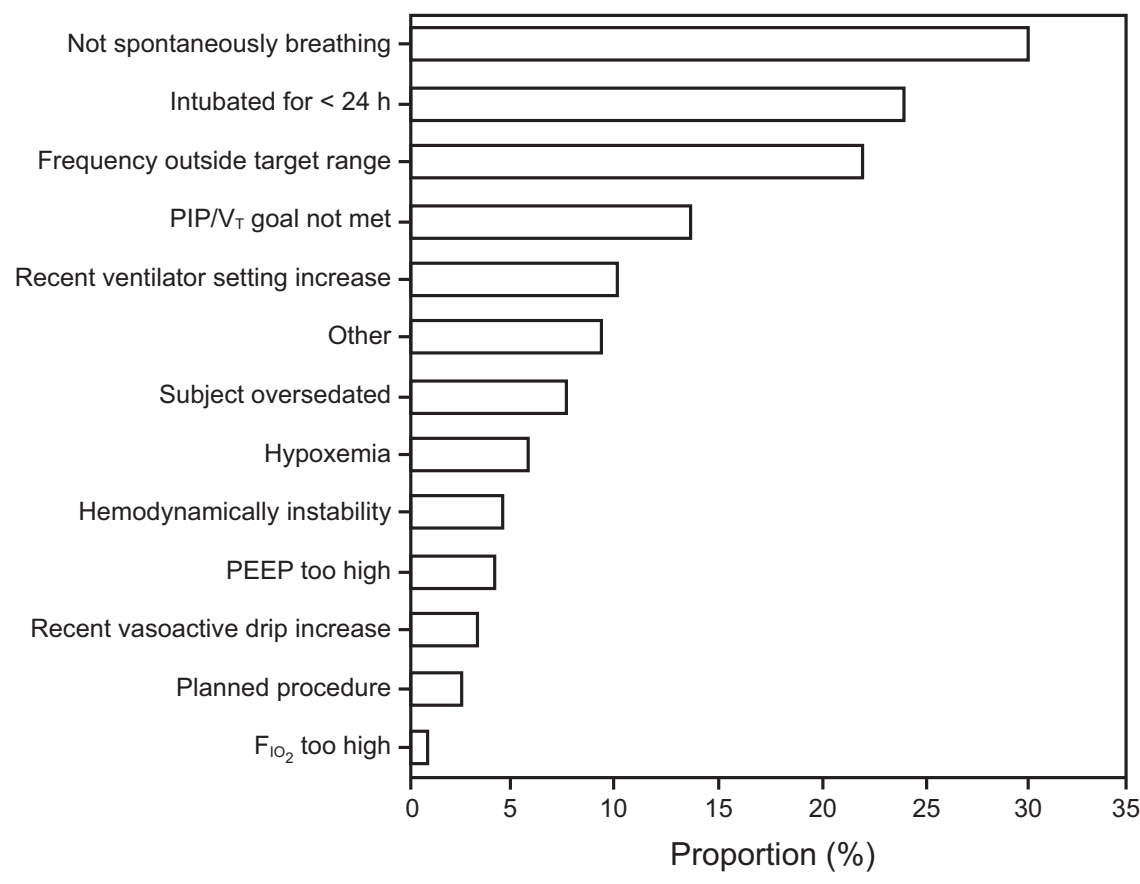

Fig. 2. Reasons for extubating "off protocol".

$57.1 \%$ of the unplanned extubation group $(4 / 7)(P<.001)$ (Table 3).

\section{Discussion}

Pediatric critical care providers lack evidence-based clinical practice guidelines for ERT compared with their adult counterparts. ${ }^{22,23}$ Therefore, it is important to continuously scrutinize extubation readiness practices and to look for further refinements to decrease both the extubation failure rates as well as the duration of invasive ventilation. This study showed that there is variation in extubation readiness practices and helped illuminate barriers to extubating patients at our institution by providing areas where efforts should be focused to further optimize our protocol.

In ideal protocolized care, all patients would be extubated per the same ERT protocol and that protocol would be flexible enough to allow for calculated deviations. A lack of spontaneous breathing was the most common reason for extubating "off protocol." This was mostly likely related to oversedation but could also be due to the neurologic status in patients with status epilepticus or new neurologic injury; these are the patients most likely to progress to a PS/CPAP trial later in the day after sedation has further 


\section{Extubation Practices in Pediatric Subjects}

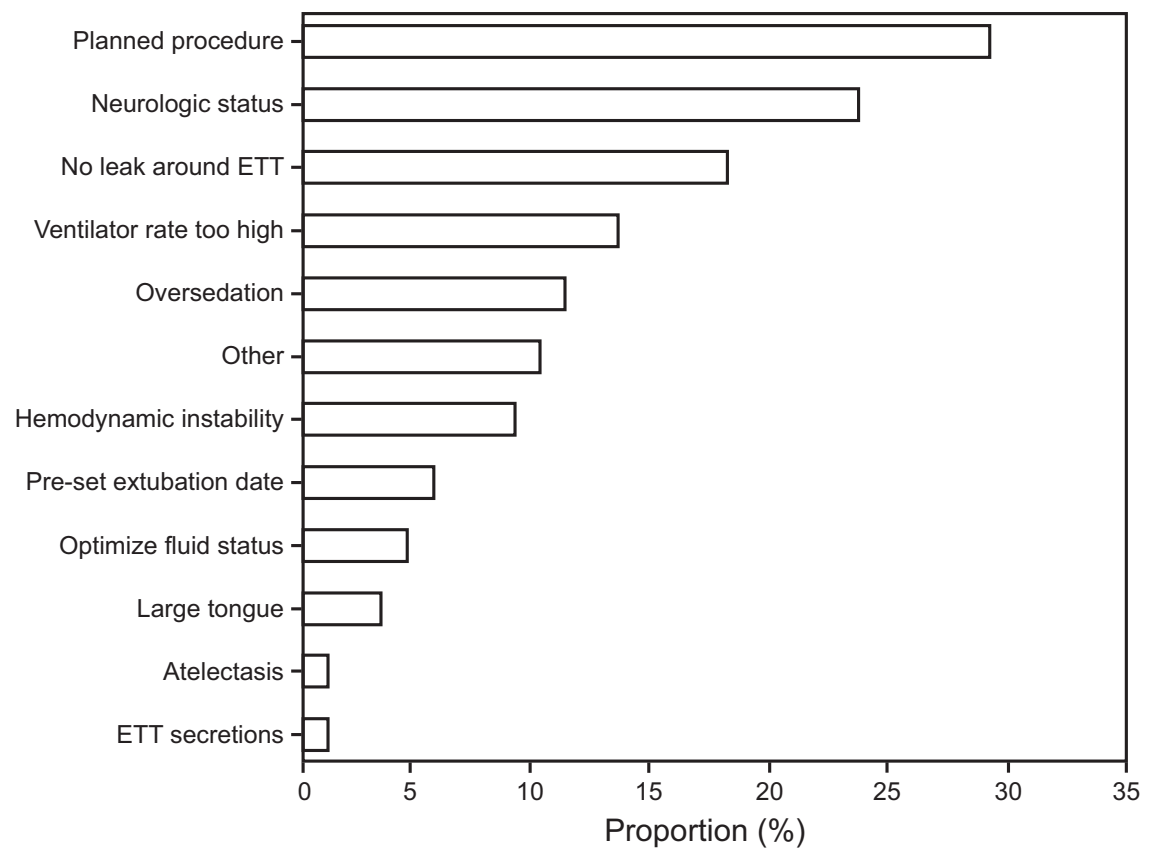

Fig. 3. Reasons to delay extubation despite passing a daily spontaneous breathing trial . ETT = endotracheal tube.

Table 3. Clinical Outcomes by Extubation Readiness Test Method

\begin{tabular}{|c|c|c|c|c|c|c|}
\hline Variable & $\begin{array}{l}\text { All Subjects } \\
(N=427)\end{array}$ & $\begin{array}{c}\text { DSBT } \\
(n=168)\end{array}$ & $\begin{array}{l}\text { PS/CPAP } \\
(n=128)\end{array}$ & $\begin{array}{l}\text { No ERT } \\
(n=124)\end{array}$ & $\begin{array}{l}\text { Unplanned Extubation } \\
\qquad(n=7)\end{array}$ & $P$ \\
\hline Initial respiratory support & & & & & & $<.001$ \\
\hline Room air & $51(11.9)$ & $11(6.6)$ & $10(7.8)$ & $30(24.2)$ & $0(0)$ & \\
\hline Nasal cannula & $221(51.8)$ & $79(47.0)$ & $69(53.9)$ & $70(56.5)$ & $3(42.9)$ & \\
\hline HFNC & $132(30.9)$ & $68(4.5)$ & 43 (33.6) & 17 (13.7) & $4(57.1)$ & \\
\hline NIV & $23(5.4)$ & $10(6.0)$ & $6(4.7)$ & $7(5.7)$ & $0(0)$ & \\
\hline Extubation failure rate & $21(4.9)$ & $15(8.9)$ & $4(3.1)$ & $0(0)$ & $2(28.6)$ & $<.001$ \\
\hline NIV use in the first $48 \mathrm{~h}$ & $25(5.9)$ & $10(6.0)$ & $7(5.5)$ & $8(6.5)$ & $0(0)$ & .91 \\
\hline \multicolumn{7}{|c|}{$\begin{array}{l}\text { Data are presented as number }(\%) \\
\text { DSBT = daily spontaneous breathing trial } \\
\text { PS = pressure support } \\
\text { ERT = extubation readiness test } \\
\text { HFNC = high-flow nasal cannula } \\
\text { NIV = noninvasive ventilation }\end{array}$} \\
\hline
\end{tabular}

been optimized. Our DSBT protocol recommends optimizing sedation and re-screening later that the day, but this practice is clinician-dependent and not currently standardized. The Society of Critical Care Medicine's ICU liberation ABCDEF bundle recommends using both spontaneous breathing trials and spontaneous awakening trials to improve patient outcomes ${ }^{24,25}$; however, spontaneous awakening trials are not a common practice in the pediatric population. Another potential solution could be incorporating sedation scoring in ventilator management protocols and ERT, or performing more frequent ERT screening.

The next most common reasons to extubate off our DSBT protocol in which an ERT was performed were breathing frequency and exhaled tidal volume outside target ranges. Bradypnea could be partially related to oversedation, whereas tachypnea and low exhaled tidal volumes could be related to residual respiratory disease, neuromuscular weakness, or undersedation. Because many subjects were extubated despite a breathing frequency above the target or a tidal volume below the target, it may be worth considering adjusting the protocol to tolerate slightly higher breathing frequencies and lower tidal volumes. ${ }^{5,26}$ The rapid shallow breathing index combines both breathing frequency and tidal volume (the rapid shallow breathing index equals breathing frequency divided by tidal volume) and has been used to predict extubation success in adults, but 


\section{Extubation Practices in Pediatric Subjects}

Table 4. Multivariate Analysis of Extubation Failure

\begin{tabular}{lcc}
\hline \hline \multicolumn{1}{c}{ Variable } & $\begin{array}{c}\text { Odds Ratio } \\
(95 \% \text { CI })\end{array}$ & $P$ \\
\hline Duration of invasive ventilation & $1.000(0.996-1.005)$ & .91 \\
Age & $0.996(0.987-1.004)$ & .31 \\
PRISM III score & $1.08(0.99-1.17)$ & .069 \\
Postoperative status & $1.46(0.42-5.07)$ & .55 \\
Primary illness category, respiratory & $8.96(2.49-32.27)$ & $<.001$ \\
Method of extubation, DSBT as & & \\
$\quad$ reference, vs & $0.33(0.10-1.12)$ & .96 \\
$\quad$ PS/CPAP & N/A & N/A \\
$\quad$ No ERT & $7.10(0.76-66.87)$ & .88 \\
$\quad$ Unplanned extubation & $0.98(0.10-9.97)$ & .99 \\
Cerebral palsy & $1.64(0.56-4.77)$ & .37 \\
Initial respiratory support after & & \\
$\quad$ extubation, HFNC & & \\
& & \\
\hline PRISM = pediatric risk of mortality & & \\
DSBT $=$ daily spontaneous breathing trial & & \\
PS = pressure support & & \\
ERT = extubation readiness test & & \\
N/A = not applicable & & \\
HFNC = high-flow nasal cannula & &
\end{tabular}

only a few pediatric studies. ${ }^{26-28}$ There is a lack of strong predictive rapid shallow breathing index values that account for different acceptable breathing frequencies in different age groups in the pediatric population. Prospective randomized controlled trials would be needed to help determine optimal pediatric rapid shallow breathing index values.

The level of PS used in the PS/CPAP group was not standardized and was provider-dependent. This reflects the lack of consensus with regard to the amount of PS required or whether PS is even needed during ERT. ${ }^{29,30}$ Although the degree of PS can conceptually affect the tidal volume and breathing frequency, a randomized controlled study is needed to explore the effects of different levels of PS on these physiologic parameters and extubation outcomes.

In the subjects who passed their DSBT but were not extubated until a future calendar day, a planned procedure was the most common documented reason. Flagging patients in the electronic medical records who have passed their DSBT could be one potential way to remind clinicians to extubate the patient after the procedure is completed, providing there are no significant changes in lung compliance, oxygenation, or hemodynamics, and patient sedationpain status allows. Protocol adjustment to allow for a repeated DSBT following procedures could be added to expedite extubation in these patients.

Neurologic status ranked as the second most common cause of extubation delay. These were subjects who either still required optimization in their sedation-pain status or who were unable to protect their airway for neurologic reasons such as status epilepticus or new neurologic injury. To minimize the proportion of patients who are oversedated, one improvement could be to incorporate sedation assessment tools, for example, the state behavioral score, or other sedation scoring tools into the protocol to assure that the patient is at the appropriate level of sedation. ${ }^{31,32}$

The utility of measuring the leak pressure around the endotracheal tube to predict upper-airway obstruction is controversial in pediatric populations. ${ }^{22,33}$ The lack of a leak was the indication for a delay in extubation for $18 \%$ of our subjects. This is also a commonly included element in ERT bundles in other studies, and it has been shown to correlate with upper-airway obstruction. ${ }^{21,34-37}$ More frequent monitoring of leak pressure, such as measuring it every shift, and the introduction of systemic corticosteroids when leak pressure is above a specific value $\left(20\right.$ or $25 \mathrm{~cm} \mathrm{H}_{2} \mathrm{O}$ ) could be incorporated into ventilator management protocols and ERT to minimize these delays. ${ }^{21,36,38}$

Although the median number of DSBTs passed before extubation was 1 regardless of the duration of invasive ventilation, the interquartile ranges demonstrate that subjects intubated for $>24 \mathrm{~h}$ passed multiple DSBTs before extubation, which may represent unnecessary delays in extubation (Table 2). Another element of delaying extubation was the time between passing any form of ERT and extubation, with a median time of $7 \mathrm{~h}$, regardless of the duration of invasive ventilation. We believe that this delay could be eliminated by the implementation of respiratory therapist-led extubation rounds as soon as patients pass an ERT, during which the respiratory therapist would extubate the patient to free up clinicians. Studies show that respiratory therapist-driven ERT pathways help improve the timeliness of ERT assessment. ${ }^{19}$

Loberger et al $^{19}$ screened pediatric subjects every $3 \mathrm{~h}$ to reduce the time between eligibility for ERT and the first ERT attempt. In their study, they were able to decrease that time from 34 to $3 \mathrm{~h}$ while simultaneously decreasing the extubation failure rate from $16 \%$ to $5 \%$. This differs from the majority of other studies that tended to evaluate subjects every 12 or 24 h. ${ }^{18,20,26}$ However, there are many challenges to a more frequent evaluation that are worth mentioning: it increases the work load on bedside respiratory therapists, it does not account for sedation optimization in preparation for extubation or provider preference to perform high-risk extubations during daytime, it will be difficult to assure nil per os status, and it might be challenging to extubate patients after midnight because this could disturb the patient's sleep cycle and reduce family satisfaction. ${ }^{19,24,39}$

Our extubation failure rate was $5 \%$ for the total cohort, which is consistent with other studies. ${ }^{8-10}$ On univariate analysis, the extubation failure rate was higher in the DSBT group (9\%) versus the PS/CPAP group (3\%). This is a rather peculiar finding, given that we hypothesized that the PS/CPAP group allowed for more permissive 


\section{Extubation Practices in Pediatric Subjects}

breathing frequencies and tidal volumes in a provider-dependent manner. Multivariate analysis showed no difference in extubation failure rates between our DSBT and PS/CPAP groups, which suggests that some barriers to extubation, such as targeted breathing frequency and tidal volume, could be relaxed yet still achieve similar extubation success. However, there is some degree of limitation in comparing this because the tidal volume and breathing frequencies were not consistently documented in our electronic medical records for the PS/CPAP group.

\section{Study Limitations and Implications}

This study was limited due to its retrospective design. The prevalence of the barriers to extubation reflect our local practices and can only be correlated with our DSBT protocol (which remained unchanged for the duration of the study). The decision to proceed to a PS/CPAP trial and the respiratory modality used after extubation reflect the clinician's preference, which was not standardized or surveyed in this study. Despite these limitations, this study demonstrated common factors that contribute to delays in extubation that providers from other institutions could use when implementing their own protocols.

\section{Conclusions}

There are variations of extubation assessment practices at our institution that could lead to delays in liberation from invasive mechanical ventilation. Adjustment to our protocol, such as tolerating higher breathing frequencies and lower tidal volumes, incorporating sedation scoring into the protocol, and implementing respiratory therapistsdriven extubation rounds, could potentially be made without significantly affecting extubation failure rates based on our findings. Further quality improvement and prospective studies would be needed to assess for improvement in time to extubation while maintaining comparable extubation failure rates.

\section{REFERENCES}

1. Khemani RG, Markovitz BP, Curley MAQ. Characteristics of children intubated and mechanically ventilated in 16 PICUs. Chest 2009;136 (3):765-771.

2. Farias JA, Fernández A, Monteverde E, Flores JC, Baltodano A, Menchaca A, et al. Mechanical ventilation in pediatric intensive care units during the season for acute lower respiratory infection: a multicenter study. Pediatr Crit Care Med 2012;13(2):158-164.

3. Slutsky AS. Lung injury caused by mechanical ventilation. Chest 1999; 116(1 Suppl):9S-15S.

4. Ricard J-D, Dreyfuss D, Saumon G. Ventilator-induced lung injury. Curr Opin Crit Care 2002;8(1):12-20.

5. Goligher EC, Dres M, Patel BK, Sahetya SK, Beitler JR, Telias I, et al. Lung- and diaphragm-protective ventilation. Am J Respir Crit Care Med 2020;202(7):950-961.
6. Khemani RG, Sekayan T, Hotz J, Flink RC, Rafferty GF, Iyer N, et al. Risk factors for pediatric extubation failure: the importance of respiratory muscle strength. Crit Care Med 2017;45(8):e798-e805.

7. Tobias JD. Tolerance, withdrawal, and physical dependency after long-term sedation and analgesia of children in the pediatric intensive care unit. Crit Care Med 2000;28(6):2122-2132.

8. Kurachek SC, Newth CJ, Quasney MW, Rice T, Sachdeva RC, Patel NR, et al. Extubation failure in pediatric intensive care: a multiplecenter study of risk factors and outcomes. Crit Care Med 2003;31 (11):2657-2664.

9. Silva-Cruz AL, Velarde-Jacay K, Carreazo NY, Escalante-Kanashiro R. Risk factors for extubation failure in the intensive care unit. Rev Bras Ter Intensiva 2018;30(3):294-300.

10. Farias JA, Alía I, Retta A, Olazarri F, Fernández A, Esteban A, et al. An evaluation of extubation failure predictors in mechanically ventilated infants and children. Intensive Care Med 2002;28(6):752-757.

11. Simonassi J, Bonora Sanso JP. Prevalence of extubation and associated risk factors at a tertiary care pediatric intensive care unit. Arch Argent Pediatr 2019;117(2):87-93.

12. Khan N, Brown A, Venkataraman ST. Predictors of extubation success and failure in mechanically ventilated infants and children. Crit Care Med 1996;24(9):1568-1579.

13. Edmunds S, Weiss I, Harrison R. Extubation failure in a large pediatric ICU population. Chest 2001;119(3):897-900.

14. Fioretto JR, Ribeiro CF, Carpi MF, Bonatto RC, Moraes MA, Fioretto EB, Fagundes DJ. Comparison between noninvasive mechanical ventilation and standard oxygen therapy in children up to 3 years old with respiratory failure after extubation: a pilot prospective randomized clinical study. Pediatr Crit Care Med 2015;16(2):124-130.

15. Yehya N. Gasping at straws: role of pressure support during spontaneous breathing trials in children. Pediatr Crit Care Med 2020;21(7):699700 .

16. Chiwane SS, Sarnaik AP. Postextubation stridor: what's all that beyond the noise? Pediatr Crit Care Med 2017;18(5):492-494.

17. Veder LL, Joosten KFM, Schlink K, Timmerman MK, Hoeve LJ, van der Schroeff MP, Pullens B. Post-extubation stridor after prolonged intubation in the pediatric intensive care unit (PICU): a prospective observational cohort study. Eur Arch Otorhinolaryngol 2020;277(6): 1725-1731.

18. Abu-Sultaneh S, Hole AJ, Tori AJ, Benneyworth BD, Lutfi R, Mastropietro CW. An interprofessional quality improvement initiative to standardize pediatric extubation readiness assessment. Pediatr Crit Care Med 2017;18(10):e463-e471.

19. Loberger JM, Jones RM, Prabhakaran P. A respiratory therapist-driven pathway improves timeliness of extubation readiness assessment in a single PICU. Pediatr Crit Care Med 2020; 21(8):e513-e521.

20. Foronda FK, Troster EJ, Farias JA, Barbas CS, Ferraro AA, Faria LS, et al. The impact of daily evaluation and spontaneous breathing test on the duration of pediatric mechanical ventilation: a randomized controlled trial. Crit Care Med 2011;39(11):2526-2533.

21. Krasinkiewicz J, Friedman ML, Slaven JE, Tori AJ, Lutfi R, AbuSultaneh S. Progression of respiratory support following pediatric extubation. Pediatr Crit Care Med. 2020. [Epub ahead of print].

22. Newth CJ, Venkataraman S, Willson DF, Meert KL, Harrison R, Dean $\mathrm{JM}$, et al. E. Weaning and extubation readiness in pediatric patients. Pediatr Crit Care Med 2009;10(1):1-11.

23. Girard TD, Alhazzani W, Kress JP, Ouellette DR, Schmidt GA, Truwit JD, et al. An Official American Thoracic Society/American College of Chest Physicians Clinical Practice Guideline: liberation from mechanical ventilation in critically ill adults. rehabilitation protocols, ventilator liberation protocols, and cuff leak tests. Am J Respir Crit Care Med 2017;195(1):120-133.

24. Pun BT, Balas MC, Barnes-Daly MA, Thompson JL, Aldrich JM, Barr J, et al. Caring for critically ill patients with the ABCDEF bundle: 


\section{Extubation Practices in Pediatric Subjects}

results of the ICU liberation collaborative in over 15,000 adults. Crit Care Med 2019;47(1):3-14.

25. Choong K, Abu-Sultaneh S. Applying the ICU liberation bundle to critically ill children. Critical Connections: The Complete News Source for Critical Care Professionals 2020;19(1):12-13.

26. Khemani RG, Hotz JC, Klein MJ, Kwok J, Park C, Lane C, et al. A phase II randomized controlled trial for lung and diaphragm protective ventilation (Real-time Effort Driven VENTilator management). Contemp Clin Trials 2020;88:105893.

27. Epstein SK. Etiology of extubation failure and the predictive value of the rapid shallow breathing index. Am J Respir Crit Care Med 1995; 152(2):545-549.

28. Cohen J, Shapiro M, Grozovski E, Fox B, Lev S, Singer P. Prediction of extubation outcome: a randomised, controlled trial with automatic tube compensation vs. pressure support ventilation. Crit Care 2009;13 (1):R21.

29. Khemani RG, Hotz J, Morzov R, Flink RC, Kamerkar A, LaFortune $\mathrm{M}$, et al. Pediatric extubation readiness tests should not use pressure support. Intensive Care Med 2016;42(8):1214-1222.

30. van Dijk J, Blokpoel RGT, Koopman AA, Dijkstra S, Burgerhof JGM, Kneyber MCJ. The effect of pressure support on imposed work of breathing during paediatric extubation readiness testing. Ann Intensive Care 2019;9(1):78.

31. Curley MA, Harris SK, Fraser KA, Johnson RA, Arnold JH. State Behavioral Scale: a sedation assessment instrument for infants and young children supported on mechanical ventilation. Pediatr Crit Care Med 2006;7(2):107-114.
32. Rosenberg L, Traube C. Sedation strategies in children with pediatric acute respiratory distress syndrome (PARDS). Ann Transl Med 2019;7(19):509.

33. Finholt DA, Henry DB, Raphaely RC. Factors affecting leak around tracheal tubes in children. Can Anaesth Soc J 1985;32(4):326-329.

34. Schneider J, Mulale U, Yamout S, Pollard S, Silver P. Impact of monitoring endotracheal tube cuff leak pressure on postextubation stridor in children. J Crit Care 2016;36:173-177.

35. Patel AB, Ani C, Feeney C. Cuff leak test and laryngeal survey for predicting post-extubation stridor. Indian J Anaesth 2015;59(2):96102.

36. Mhanna MJ, Anderson IM, Iyer NP, Baumann A. The use of extubation readiness parameters: a survey of pediatric critical care physicians. Respir Care 2014;59(3):334-339.

37. Khemani RG, Hotz J, Morzov R, Flink R, Kamerkar A, Ross PA, Newth CJL. Evaluating risk factors for pediatric post-extubation upper airway obstruction using a physiology-based tool. Am J Respir Crit Care Med 2016;193(2):198-209.

38. Baranwal AK, Meena JP, Singhi SC, Muralidharan J. Dexamethasone pretreatment for $24 \mathrm{~h}$ versus $6 \mathrm{~h}$ for prevention of postextubation airway obstruction in children: a randomized double-blind trial. Intensive Care Med 2014;40(9):1285-1294.

39. Burns KEA, Wong JTY, Dodek P, Cook DJ, Lamontagne F, Cohen A, et al. Frequency of screening for weaning from mechanical ventilation: two contemporaneous proof-of-principle randomized controlled trials. Crit Care Med 2019;47(6):817-825. 